Regular Article -clinical research

\title{
Functional Liver Reserve Parameters Predictive for Posthepatectomy
}

\section{Complications}

\author{
Atsushi Nanashima, MD, ${ }^{1}$ Takafumi Abo, MD, ${ }^{1}$ Junichi Arai, MD, ${ }^{1}$ \\ Hirofumi Matsumoto, MD, ${ }^{1}$ Takashi Kudo, MD, ${ }^{2}$ and Takeshi Nagayasu MD ${ }^{1}$ \\ ${ }^{1}$ Division of Surgical Oncology and Department of Surgery, and ${ }^{2}$ Department of \\ Radioisotope Medicine, Nagasaki University Graduate School of Biomedical \\ Sciences, 1-7-1 Sakamoto, Nagasaki, 8528501, JAPAN
}

Running title: Liver function parameters predictive of complications

Correspondence to: Atsushi Nanashima, MD,

Division of Surgical Oncology, Department of Surgery, Nagasaki University Graduate School of Biomedical Sciences, 1-7-1 Sakamoto, Nagasaki 852-8501, JAPAN

Tel: +81-95-849-7304 Fax: +81-95-849-7306

E-mail: a-nanasm@nagasaki-u.ac.jp

This study was undertaken without any external financial support. 


\section{Abstract.}

Background: Posthepatectomy complications have markedly decreased with advances in techniques and management; however, surgical risk to patients with injured livers is still not negligible. We evaluated several preoperative parameters of functional liver reserve tests in patients with various liver diseases as predictors of posthepatectomy complications. A comprehensive evaluation of preoperative liver functions is necessary for the prediction of the risk of posthepatectomy complications. Methods: Over a 10-year period, we examined 442 patients who underwent hepatectomy for liver and biliary diseases. The patients’ background liver conditions included chronic viral liver diseases in 211 patients, obstructive jaundice in 29 patients and normal liver in 202. Hepatectomy-related postoperative complications (i.e., long-term ascites, intra-abdominal infection and hepatic failure) occurred in 115 (26\%) patients. A multivariate logistic analysis was performed to detect the predictive parameters, and a multivariate linear regression analysis was performed to derive a predictive formula for complications. Results: univariate analysis identified 15 significant parameters associated with hepatectomy-related complications, and 8 parameters were independently predictive factors identified in the multivariate analysis (presence of chronic hepatic injury, clearance index (HH15) by technetium- ${ }^{99 m}$ galactosyl serum albumin scintigraphy of $\geq 0.60$, total bilirubin level $>1 \mathrm{mg} / \mathrm{dl}$, serum hyaluronic acid (HA) level $\geq 75 \mathrm{ng} / \mathrm{ml}$, major hepatectomy, blood loss $\geq 950 \mathrm{ml}$, operating 
time $\geq 500$ minutes, and combined resection of another organ or major vessel). HH15, bilirubin level, HA, and major hepatectomy were parameters included in the predictive formula. Conclusions: in this study, we present a comprehensive formula based on predictive parameters for hepatic complications for prospective assessment to avoid post-hepatectomy morbidity.

Keywords: liver resection, prognostic functional parameters, posthepatectomy complications, 99mTc-GSA liver scintigraphy. 


\section{Introduction}

The operative morbidity and mortality rates in patients with liver diseases who underwent hepatic resection have markedly decreased in the recent years due to advances in operative techniques and improvements in perioperative management [1]. However, in patients with liver dysfunction, such as cirrhosis and obstructive jaundice, and in those who undergo extended major hepatectomy, the operative risk is significant and should be carefully evaluated before surgery [2, 3]. Hyperbilirubinemia, uncontrolled ascites, and intra-abdominal infection are severely troublesome complications after hepatectomy that can decrease patient quality of life, prolong hospitalization and cause life-threatening hepatic failure [4]. To reduce the incidence of such postoperative hepatic complications, careful preoperative planning and patient selection should involve an analysis of complication-associated parameters of liver function tests, histological examination and surgical records.

Indocyanine green (ICG) has been used to evaluate hepatic functional reserve before hepatectomy and provides useful information for clinical decision-making regarding the indications or extent of hepatic resection in patients with injured livers [5-9]. Miyagawa et al. proposed the Makuuchi's criteria for the range of hepatectomy by ICG retention rate at 15 minutes (ICGR15), the serum level of total bilirubin and the presence of ascites [5]. Although these criteria have been extremely useful in Japan, in some patients with liver damage or obstructive jaundice, surgery may be considered outside of the criteria. Therefore, advanced 
criteria including additional reliable functional parameters were needed. Recently, new tests of hepatic functional reserve, such as serum hyaluronic acid (HA) level [10], 99 mTc-galactosyl human serum albumin liver scintigraphy (99 mTc-GSA scintigraphy) [11], and interleukin levels [12] have been used to evaluate post-hepatectomy complications. Although several recent studies including our own have attempted these analyses to predict complications, [14-16] no distinct criteria or final consensus using these parameters have been widely accepted [17]. In an effort to better define the factors that could predict postoperative hepatic complications or failure, a comprehensive analysis of reliable functional parameters is necessary.

In the present study, we examined the relationship between the abovementioned reliable parameters of functional liver reserve and post-hepatectomy complications in 442 patients with various liver diseases who underwent hepatectomy. Furthermore, we attempted to derive a comprehensive analytical formula through multivariate analysis for the prediction of hepatic complications. 


\section{Materials and Methods}

\subsection{Patients}

The subjects were 442 patients with liver diseases who underwent hepatectomy in the Division of Surgical Oncology, Department of Surgery in Nagasaki University Graduate School of Biomedical Sciences between 2001 and January 2013. They included 299 men and 143 women with a mean age of $66 \pm 11$ years $( \pm S D$, range, 24-86 years). The liver diseases warranting hepatic resection included hepatocellular carcinomas in 197 patients, metastatic liver carcinomas in 123, intrahepatic cholangiocarcinomas in 44 , gallbladder carcinomas in 18 , bile duct carcinomas in 40 and other liver diseases in 20. The background liver diseases included chronic viral hepatitis in 284 patients including cirrhosis in 73 (hepatitis B in 102 patients (36\%), hepatitis C in 159 (56\%) and both hepatitis B and C in 23(8\%)), obstructive jaundice in 29 patients (including bile duct carcinomas in 24 patients (83\%), intrahepatic cholangiocarcinomas in three (11\%), gallbladder carcinomas in one (3\%) and hepatocellular carcinoma in one (3\%)) and normal liver in 202.

In our hospital, the volume of liver to be resected is estimated before surgery based on the results of the indocyanine green retention rate at 15 min (ICG R15), using Takasaki’s formula [18]. The liver volume, excluding any tumor volume $\left(\mathrm{cm}^{3}\right)$ is measured by computed tomography (CT) volumetry [19]. Since 2004, we have modified our criteria and applied ${ }^{99 \mathrm{~m}}$ Tc-GSA scintigraphy and its functional hepatic volumetry, serum HA level and prothrombin activity in addition to the 6 
ICGR15 test to decide the extent of hepatectomy [16, 20]. In this cohort, we performed limited resection in 124 patients, subsegmentectomy or segmentectomy in 134 , lobectomy in 98 , and extended lobectomy in 86 . Postoperative hepatectomy-related complications occurred in 115 (26\%) patients and included persistent ascites (defined as massive ascites unresponsive to diuretics for more than 2 weeks) in 78 patients; intra-abdominal infection or abscess formation in 40 patients; and hepatic failure (defined by total bilirubin of $>5 \mathrm{mg} / \mathrm{dl}$ on postoperative day (POD) 7 or postoperative death without other cause) in 23 patients. Death during hospital stay was observed in 8 patients (2\%). Laparoscopic limited resection or left lateral sectionectomy was performed in 18 patients (4\%). Combined resection of other organs (including colon in 42 (37\%), pancreas in 18 (16\%), spleen in 16 (14\%), stomach in $11(9 \%)$, diaphragm in $10(8 \%)$, rectum in seven (6\%), adrenal gland in four (3\%), duodenum in two (2\%), lung in two (2\%), ovary in two (2\%) and kidney in one (1\%)), was performed in 115 (26\%). Combined resection of bile ducts with a biliary anastomosis (including bile duct carcinoma in 39 patients (46\%), intrahepatic cholangiocarcinomas in 17 (20\%), gallbladder carcinomas 14 (16\%), liver metastasis in five (6\%), hepatocellular carcinomas in five (6\%), hepatolithiasis in four (5\%) and necroendocrine tumor in one (1\%) was performed in 85 (19\%). No biliary anastomosis leakage was observed in the present series. Combined resection of major vessels with an anastomosis (including portal vein in 27 patients (59\%), hepatic artery in seven (15\%), hepatic veins in six (13\%) and vena cava in six (13\%)) was performed in 46 
patients (10\%), and no postoperative occlusion was observed. The study design was approved by the Ethics Review Board of our university, and informed consent for data collection was obtained for each patient.

\subsection{Evaluated parameters}

Clinical data, conventional liver functions and surgical data were analyzed. ICG was injected intravenously at a dose of $0.5 \mathrm{mg} / \mathrm{kg}$ body weight, and the 15 -minute retention rate was measured by a photopiece applied to the fingertip (RK-1000, Sumitomo Electric, Tokyo, Japan) without blood sampling [16, 20].

Patients received $3 \mathrm{mg}(185 \mathrm{MBq})$ of ${ }^{99 \mathrm{~m}}$ Tc-GSA (Nihon Medi-Physics, Nishinomiya, Japan) as a bolus dose into the antecubital vein. The clearance index of ${ }^{99 \mathrm{~m}}$ Tc-GSA (HH15) and the hepatic uptake ratio of ${ }^{99 \mathrm{~m}}$ Tc-GSA (i.e., LHL15; the count ratio of the liver to the sum of the heart and liver 15 min after injection of 99 mTc-GSA) were calculated after injection of ${ }^{99 \mathrm{~m}}$ Tc-GSA [11, 20]. We calculated the ratio of LHL15 and HH15 (LHL/HH15) in the present study.

Peripheral blood samples for serum HA level assays were collected from every patient in stable condition early in the morning during hospitalization. Blood samples were centrifuged at $3000 \mathrm{rpm}$ for $15 \mathrm{~min}$, and serum was stored at $-80^{\circ} \mathrm{C}$. HA was assayed using the sandwich protein-binding assay (SRL, Inc., Tokyo, Japan). The normal serum HA level determined by the laboratory of SRL, Inc. is $<50$ ng/ml. [20] 
We used the Liver Damage Grade using ICGR15 instead of encephalopathy in the five parameters of Child-Pugh classification of the Liver Cancer Study Group of Japan in 2000 [21]. The conventional liver functional parameters, including total bilirubin level, prothrombin activity, platelet count and alanine aminotransferase level were examined. Surgical records, including blood loss, blood transfusion, and operative and transection time under hepatic inflow occlusion were examined.

Role of authors was as follows: Dr A.N. as a main author organizing the entire research, Dr. T.A., Dr. J. A, and Dr. H.M. as the staff members of the liver surgery group who collected data of liver functions, Prof. T.K. as an expert of nuclear medicine regarding liver scintigraphy who measured LHL15 and HH15, and Prof. T.N. as a supervisor who managed all clinical researches in the Department of Surgery.

\subsection{Statistical analysis}

Continuous data are expressed as means \pm SD. Data were compared between groups using one-way analysis of variance (ANOVA) and were examined with the Mann-Whitney U-test. The optimal predictive cut-off value for post-hepatectomy complications was determined for each parameter by the analysis of receiver operating characteristic (ROC) curves. Comparisons of categorical data were performed with the Chi-square test. Logistic regression analysis was performed to determine the predictive value of risk factors. Potentially predictive variables were 9 
identified using a significance level of $\mathrm{p}<0.05$ by univariate analysis, and the identified factors were then entered into a logistic regression multivariate analysis. A two-tailed $P$ value $<0.05$ was considered significant. The Statistical Package for the Social Sciences (SPSS) version 18.0 software (SPSS, Chicago, IL) was used for all statistical analyses. 


\section{Results}

\subsection{Relationship between associated parameters and post-hepatectomy complications}

Table 1 shows the comparisons of 14 continuous variables between patients with and without post-hepatectomy complications. Univariate analysis identified 10 variables that were significantly different between patients with and without post-hepatectomy complications ( $\mathrm{p}<0.05)$, including ICGR15, LHL15, HH15, LHL15/HH15, total bilirubin level, prothrombin activity, serum HA level, operating time, intraoperative blood loss and blood transfusion. The predictive cut-off values for post-hepatectomy complications for each of these parameters were calculated by the analysis of their ROC curves.

Table 2 shows the comparisons of 8 categorical variables between patients with and without post-hepatectomy complications. Univariate analysis identified that the distribution of chronic hepatic injuries or grade B liver damage was significantly different between patients with and without post-hepatectomy complications $\left(\mathrm{p}^{<0.01)}\right.$. Furthermore, major hepatectomy and the presence of combined resections of other organ or major vessels were significantly more frequent in patients with complications than in those without $(\mathrm{p}<0.05)$.

\subsection{Multivariate analysis of associated factors and predictive formula for post-hepatectomy complications}


Among the above 15 parameters, blood loss and transfusion could not be preoperatively predicted and the Liver Damage Grade included other comparable parameters. Therefore, the remaining 12 clinicopathological and surgical parameters were entered into the multivariate analysis to define a logistic regression model of associations with post-hepatectomy complications. With respect to total hepatectomy-related complications, the multivariate analysis identified the presence of chronic hepatic injury, HH15, total bilirubin level, serum HA level and the extent of hepatectomy as five significant, independent factors associated with post-hepatectomy complications (Table 3). For the prediction of hepatic failure, HH15, the extent of hepatectomy and operating time were significant independent predictors. For uncontrolled ascites, the presence of chronic hepatic injury, total bilirubin level, serum HA level and the combined resection of other organs were significant independent predictors. For intra-abdominal infection, only the operative time and the combined resection of other organs were significant independent predictors.

The selected hepatic parameters were correlated with the total hepatic complications using a binomial logistic regression analysis with the forced entry method (Table 4). Based on the multivariate logistic analysis, HH15, total bilirubin level, serum HA levels and the extent of hepatectomy correlated significantly with hepatic complications. Based on this result, the regression equation was established using $\exp (\beta)$ as: 
$\mathrm{Y}$ (presence of post-hepatectomy complications $)=1.734 \times(1$ for HH15 $\geq 0.6$, or 0 for others) $+2.468 \times$ ( 1 for total bilirubin level $>1 \mathrm{mg} / \mathrm{dl}$, or 0 for others $)+1.833$ $\mathrm{x}$ ( 1 for serum HA level $>75 \mathrm{ng} / \mathrm{ml}, 0$ for others $)+2.523 \mathrm{x}$ ( 1 for major hepatectomy (hemihepatectomy or more), or 0 for others) +0.088 .

\subsection{Prediction of post-hepatectomy complication by the predictive formula}

Based on the ROC analysis of this formula, the risk of any hepatectomy-related complication would be considered substantial for $Y$ values $\geq 2.5$. (Fig. 1a)

With respect to uncontrolled ascites predicted by the same analysis (Table 5), the regression equation was established as:

$\mathrm{Y}($ uncontrolled ascites $)=1.936 \times(1$ for the presence of chronic hepatic injury, or 0 for others $)+1.818 \times$ ( 1 for total bilirubin level $>1 \mathrm{mg} / \mathrm{dl}$, or 0 for others $)+2.736 \mathrm{x}$ ( 1 for serum HA level $>75 \mathrm{ng} / \mathrm{ml}, 0$ for others) $+2.214 \times$ ( 1 for the combined resection of other organs, or 0 for others) +0.062 . Based on the ROC analysis of this formula, the risk of uncontrolled ascites would be considered substantial for Y values $\geq 3.0$ (Fig. 1b).

For intra-abdominal infection prediction by the same analysis (Table 5), the regression equation was established as:

$\mathrm{Y}($ intra-abdominal infection $)=2.344 \times(1$ for combined vascular resection, or 0 for others $)+7.381 \times$ ( 1 for operative time $>500$ minutes, or 0 for others $)+0.022$. Based on the ROC analysis of this formula, the risk of intra-abdominal infection would be considered substantial for $Y$ values $\geq 5.7$ (Fig. 1c). 
For the prediction of hepatic failure by the same analysis (Table 5), the regression equation was established as:

$\mathrm{Y}($ hepatic failure $)=4.715 \times(1$ for HH15 $>0.60$, or 0 for others $)+5.734 \times(1$ for operative time $>500$ minutes, or 0 for others $)+0.005$. Based on the ROC analysis of this formula, the risk of hepatic failure would be considered substantial for $\mathrm{Y}$ values $\geq 7.2$ (Fig. 1d). 


\section{Discussion}

Previous investigators have reported various risk factors associated with post-hepatectomy complications as described above, including the presence of cirrhotic liver or severe steatohepatitis [3, 4, 22]; liver functional parameters such as ICG clearance, liver scintigraphy, serum bilirubin level, platelet counts, galactose elimination capacity, prothrombin activity, $\gamma$-glutamyltranspeptidase, serum HA level, arterial blood ketone body ratio, hepatocyte growth factor, [2, 10, 11, 23-27], intraoperative portal pressure [28]; and surgical parameters including the remnant liver volume, hepatic inflow occlusion, the amount of blood loss or transfusion or the surgeon's level of experience [29-33]. By considering the above factors, the surgeon carefully decides upon the indication of hepatectomy and the extent of resected volume to avoid excessive hepatectomy or lethal postoperative morbidities, such as hepatic failure. We must always consider an adequate balance between curability of the malignant tumor and surgical risk factors. This balance is sometimes difficult to decide in patients with borderline liver functions due to liver damage. Recently, in patients with poor liver functions with small liver tumors of less than $2 \mathrm{~cm}$, non-surgical modalities, such as ablation therapy, have been applied with improved curability or prognosis [34]. Therefore, the necessity for aggressive limited hepatectomy for such small lesions has been decreased. However, in cases of very large liver tumors or advanced biliary carcinomas, a large volume of hepatectomy is still necessary, and liver functions are often poor due to chronically injured liver or obstructive jaundice. [35, 36] In such cases, 15 
surgeons should examine the more important or reliable predictive parameters before conducting hepatectomy. We previously attempted to apply various liver functional reserve indicators, including serum HA level and 99 mTc-GSA scintigraphy based on the ICG R15 test, which might improve patient outcomes and lead to spread operative indications [16, 20, 37]. However, we were unable to achieve a distinct, comprehensive strategy for determining the indication of hepatectomy for patients with a background of various liver diseases thus far. In the present study, to address this issue, we further examined the relationship between significantly predictive factors and post-hepatectomy morbidities in a large series of patients collected over the last 10 years.

In this study, we analyzed the relationship of 22 parameters of patient demographics, hepatic functional parameters or surgical results with three major hepatectomy-related complications. Similar to previous reports [2-12, 16, 20, 23, 29, 30], the 15 parameters significantly associated with total hepatic complications were selected by univariate analysis. In the present analysis, we considered the significance of HH15 and of the ratio between LHL15 and HH15, although HH15 has not been well evaluated previously. However, a few reports showed the significance of HH15 for the specific evaluation of liver function [38, 39], and we have experienced that an increased HH15 may predict poor surgical outcomes. Furthermore, a new surgical device, the vessel sealer, has recently been applied in the field of hepatic transection, and this advance may reduce the operative blood loss. [40] Therefore, we additionally examined certain operative parameters, 16 
including the use of a hemostatic device, in the present study. However, the use of such a device did not significantly contribute to postoperative liver dysfunction. The present multivariate analysis identified several parameters which were significantly associated with posthepatectomy complications, but not ICGR15, LHL15 or prothrombin activity, as in our previous study [16, 20]. Because these parameters were already applied in the decision of operative indication, their data may not reflect clinical outcomes. Instead, HH15, total bilirubin level and serum HA level were associated with morbidities.

The extent of hepatectomy was also a significant risk factor for posthepatectomy complications despite the fact that the operative indication has been distinctly controlled recently [16, 20]. Therefore, it is necessary to use additional parameters improve our indication criteria in addition to improvement of surgical techniques. In the present study, we examined the correlations for each complication individually and found that the related parameters were different for each complication. With respect to intra-abdominal infection, surgical parameters were significant predictors, but liver functions were not. In cases of combined resections accompanied by hepatectomy, it is necessary to take measures to prevent infections, such improvements of surgical techniques or the induction of immunonutrition with synbiotics [41]. With respect to uncontrolled ascites or hepatic failure, both preoperative liver functions and surgical parameters were well correlated, as previously reported [42]. 
In the next step, we attempted to derive a predictive formula for each complication for preoperative assessment. The amount of blood loss or transfusion could not be accurately predicted before operation, and the Liver Damage Grade included another three predictive parameters of ICGR15, bilirubin level and prothrombin activity [43]. These parameters were therefore not applied in the next multivariate linear regression analysis. To reduce blood loss and transfusion, the improvement of surgical skills or greater utilization of auto-transfusion were necessary, as previously reported [40,44]. The Liver Damage Grade according to the Liver Cancer Study Group of Japan [21, 43] may be useful as a comprehensive liver functional scoring system for the prediction of posthepatectomy complications. HH15 and serum HA level correlated with complications in the present study. The serum HA concentration reflects the function of hepatic sinusoids and is a sensitive marker of liver endothelial cell dysfunction after liver ischemia [45, 46]. Yachida et al. [10] and we in our previous study [37] reported a predictive cut-off value of $150 \mathrm{ng} / \mathrm{ml}$ for post-hepatectomy complications. However, in the present study, a lower cut-off level of $75 \mathrm{ng} / \mathrm{ml}$ was useful for the prediction of various complications. As expected by our hypothesis, HH15 was also significantly correlated with posthepatectomy complications. Even if LHL15 level is normal, it is necessary to consider the functional liver reserve when HH15 is increased over 0.6 , according to our present results. We attempted to complete the predictive formula; however, a close correlation with morbidity was not 
observed at this stage. Therefore, we should prospectively apply these formulae in the next validation set of patients to clarify the significance of our present trial.

In the present study, the relationships between 22 clinicopathologic/surgical parameters and post-hepatectomy complications were examined. Our results showed that 15 parameters were significantly associated with post-hepatectomy complications by the univariate analysis and 8 parameters (the presence of chronic hepatic injury, HH15, total bilirubin level, serum HA level, the extent of hepatectomy, the combined resection of another organ or major vessels accompanied with hepatectomy and operative time) were selected by the multivariate analysis. Increased HH15, total bilirubin level, serum HA, and major hepatectomy were independent predictive parameters for overall hepatectomy-related complications. The presence of chronic liver injury, increased levels of bilirubin and serum HA predicted uncontrolled ascites; the combined resection of major vessels and longer operative time predicted intra-abdominal infection; and increased levels of HH15 and serum HA predicted hepatic failure. This comprehensive predictive model should be useful even in posthepatectomy patients with chronic viral hepatitis and bile duct carcinomas [47].

In conclusion, in patients who are scheduled for major hepatic resection, certain pretreatments, such as portal embolization or reduced scope of surgery, are useful to avoid posthepatectomy complications, and efforts to limit blood loss or transfusion during surgery should also be considered in planning the technical aspects of surgical management. The comprehensive predictive formulae from the 19 
present analysis would be useful for the prediction of morbidity before hepatectomy, and their further prospective evaluation is necessary. Through an adequately comprehensive assessment of functional and surgical factors, more appropriate treatment strategies may be selected for patients with hepatic injuries to avoid serious posthepatectomy complications. 


\section{REFERENCES}

(1) Poon RT, Fan ST, Lo CM, et al. Improving perioperative outcome expands the role of hepatectomy in management of benign and malignant hepatobiliary diseases: analysis of 1222 consecutive patients from a prospective database. Ann Surg. 2004;240:698.

(2) Kokudo N, Vera DR, Tada K, et al. Predictors of successful hepatic resection: Prognostic usefulness of hepatic asialoglycoprotein receptor analysis. World J Surg. 2002;26:1342.

(3) Fan T, Lo CM, Liu CL, et al. Hepatectomy for hepatocellular carcinoma: toward zero hospital deaths. Ann. Surg. 1999;229: 322.

(4) Fan ST. Methods and related drawbacks in the estimation of surgical risks in cirrhotic patients undergoing hepatectomy. Hepatogastroenterology 2002;49:17.

(5) Miyagawa S, Makuuchi M, Kawasaki S, et al. Criteria for safe hepatic resection. Am. J Surg. 1995;169: 589.

(6) Hemming AW, Scudamore CH, Shackleton CR, et al. Indocyanine green clearance as a predictor of successful hepatic resection in cirrhotic patients. Am. J Surg. 1992;163: 515.

(7) Nagino M, Nimura Y, Kamiya J, et al. Changes in hepatic lobe volume in biliary tract cancer patients after right portal vein embolization. Hepatology. 1995;21:434. 
(8) El-Desoky A, Seifalian AM, Cope M, et al. Experimental study of liver dysfunction evaluated by direct indocyanine green clearance using near infrared spectroscopy. Br J Surg. 1999;86:1005.

(9) Schneider PD. Preoperative assessment of liver function. Surg Clin North Am. 2004;84:355.

(10) Yachida S, Wakabayashi H, Kokudo Y, et al. Measurement of serum hyaluronate as a predictor of human liver failure after major hepatectomy. World J Surg. 2000;24:359.

(11) Mitsumori A, Nagaya I, Kimoto S, et al. Preoperative evaluation of hepatic functional reserve following hepatectomy by technetium-99 m galactosyl human serum albumin liver scintigraphy and computed tomography. Eur. J Nucl. Med. 1998;25:1377.

(12) Yamada T, Hisanaga M, Nakajima Y, et al. The serum interleukin 8 level reflects hepatic mitochondrial redox state in hyperthermochemohypoxic isolated liver perfusion with use of a venovenous bypass. Surgery. 1999;125:304.

(13) Andres A, Toso C, Moldovan B, et al. Complications of elective liver resections in a center with low mortality: a simple score to predict morbidity. Arch Surg. 2011;146:1246.

(14) Cucchetti A, Ercolani G, Vivarelli M, et al. Impact of model for end-stage liver disease (MELD) score on prognosis after hepatectomy for hepatocellular carcinoma on cirrhosis. Liver Transpl. 2006;12:966. 
(15) Imamura H, Sano K, Sugawara Y, et al. Assessment of hepatic reserve for indication of hepatic resection: decision tree incorporating indocyanine green test. J Hepatobiliary Pancreat Surg. 2005;12:16.

(16) Nanashima A, Tobinaga S, Abo T, et al. Reducing the incidence of post-hepatectomy hepatic complications by preoperatively applying parameters predictive of liver function. J Hepatobiliary Pancreat Sci. 2010;17:871.

(17) Balzan S, Belghiti J, Farges O, et al. The "50-50 criteria" on postoperative day 5: an accurate predictor of liver failure and death after hepatectomy. Ann Surg. 2005;242:824.

(18) Takasaki T, Kobayashi S, Suzuki S, et al. Predetermining postoperative hepatic function for hepatectomies. Int. Surg. 1980;65:309.

(19) Kubota K, Makuuchi M, Kusaka K, et al. Measurement of liver volume and hepatic functional reserve as a guide to decision-making in resection surgery for hepatic tumors. Hepatology 1997;26:1176.

(20) Nanashima A, Yamaguchi H, Shibasaki S, et al. Relationship between indocyanine green test and technetium-99 m galactosyl serum albumin scintigraphy in patients scheduled for hepatectomy: Clinical evaluation and patient outcome. Hepatol Res. 2004;28:184.

(21) Liver cancer study group of Japan. G. Stage. In, editor, Makuuchi M, ed. General Rules for the Clinical and Pathological Study of Primary Liver Cancer The second English edition. Tokyo, Kanehara \& Co. 2003:23-25. 
(22) Reddy SK, Marsh JW, Varley PR, et al. Underlying steatohepatitis, but not simple hepatic steatosis, increases morbidity after liver resection: a case-control study. Hepatology. 2012;56:2221.

(23) Ishigami Y, Masuzawa M, Miyoshi E, et al. Clinical applications of ICG finger monitor in patients with liver disease. J Hepatol. 1993;19:232.

(24) Hwang EH, Taki J, Shuke N, et al. Preoperative assessment of residual hepatic functional reserve using 99 mTc-DTPA-galactosyl-human serum albumin dynamic SPECT. J Nucl. Med. 1999;40:1644.

(25) Fujii Y, Shimada H, Endo I, et al. Risk factors of posthepatectomy liver failure after portal vein embolization. J Hepatobiliary Pancreat. Surg. 2003;10:226.

(26) Redaelli C, Dufour JF, Wagner M, et al. Preoperative Galactose elimination capacity predicts complications and survival after hepatic resection. Ann. Surg. 2002;235:77.

(27) Yoshikawa A, Kaido T, Seto S, et al. Hepatocyte growth factor promotes liver regeneration with prompt improvement of hyperbilirubinemia in hepatectomized cholestatic rats. J Surg. Res. 1998;78:54.

(28) Chen X, Zhai J, Cai X, et al. Severity of portal hypertension and prediction of postoperative liver failure after liver resection in patients with Child-Pugh grade A cirrhosis. Br J Surg. 2012;99:1701.

(29) Greenburg AG. Benefits and risks of blood transfusion in surgical patients. World J Surg. 1996;20:1189. 
(30) Shirabe K, Shimada M, Gion T, et al. Postoperative liver failure after major hepatic resection for hepatocellular carcinoma in the modern era with special reference to remnant liver volume. J Am. Coll. Surg. 1999;188:304.

(31) Yanaga K, Honda H, Ikeda Y, et al. Significance of liver size in hepatic surgery. HPB Surg. 1997;10:195.

(32) Makuuchi M, Thai BL, Takayasu K, et al. Preoperative portal embolization to increase safety of major hepatectomy for hilar bile duct carcinoma: a preliminary report. Surgery 1990;107:521.

(33) Dufour DR, Lott JA, Nolte FS, et al. Diagnosis and monitoring of hepatic injury. II. Recommendations for use of laboratory tests in screening, diagnosis, and monitoring. Clin. Chem. 2000;46:2050.

(34) N'Kontchou G, Mahamoudi A, Aout M, et al. Radiofrequency ablation of hepatocellular carcinoma: long-term results and prognostic factors in 235 Western patients with cirrhosis. Hepatology. 2009;50:1475.

(35) Sakamoto Y, Shimada K, Nara S, et al. Risk factors for early bilirubinemia after major hepatectomy for perihilar cholangiocarcinoma with portal vein embolization. Hepatogastroenterology. 2010;57:22.

(36) Chen XP, Qiu FZ, Wu ZD, et al. Chinese experience with hepatectomy for huge hepatocellular carcinoma. Br J Surg. 2004;91:322.

(37) Nanashima A, Yamaguchi Y, Tanaka K, et al. Preoperative serum hyaluronic acid level as a good predictor of posthepatectomy complications. Surg. Today 2004;34:913. 
(38) Kawamura H, Kamiyama T, Nakagawa T, et al. Preoperative evaluation of hepatic functional reserve by converted ICGR15 calculated from Tc-GSA scintigraphy. J Gastroenterol Hepatol. 2008;23:1235.

(39) Iguchi T, Sato S, Kouno Y, et al. Comparison of Tc-99 m-GSA scintigraphy with hepatic fibrosis and regeneration in patients with hepatectomy. Ann Nucl Med. 2003;17:227.

(40) Chiappa A, Zbar AP, Bertani E, et al. The Ligasure vessel sealer in liver resection: a pilot study. Hepatogastroenterology. 2007;54:2353.

(41) Sugawara G, Nagino M, Nishio H, et al. Perioperative synbiotic treatment to prevent postoperative infectious complications in biliary cancer surgery: a randomized controlled trial. Ann Surg. 2006;244:706.

(42) Yang T, Zhang J, Lu JH, et al. Risk factors influencing postoperative outcomes of major hepatic resection of hepatocellular carcinoma for patients with underlying liver diseases. World J Surg. 2011;35:2073.

(43) Ikai I, Takayasu K, Omata M, et al; Liver Cancer Study Group of Japan. A modified Japan Integrated Stage score for prognostic assessment in patients with hepatocellular carcinoma. J Gastroenterol. 2006;41:884.

(44) Park JO, Gonen M, D'Angelica MI, et al. Autologous versus allogeneic transfusions: no difference in perioperative outcome after partial hepatectomy. Autologous transfusion on hepatectomy outcome. J Gastrointest Surg. 2007;11:1286. 
(45) Pontinha N, Pessegueiro H, Barros H. Serum hyaluronan as a marker of liver fibrosis in asymptomatic chronic viral hepatitis B. Scand. J Clin. Lab. Invest. 1999;59:343.

(46) Idobe Y, Murawaki Y, Ikuta Y, et al. Post-prandial serum hyaluronan concentration in patients with chronic liver disease. Intern Med. 1998;37:568.

(47) Das BC, Isaji S, Kawarada Y. Analysis of 100 consecutive hepatectomies: risk factors in patients with liver cirrhosis or obstructive jaundice. World J Surg. 2001;25:266. 


\section{FIGURE LEGEND}

Fig. 1 The analysis of receiver operating characteristic (ROC) curves using each predictive formula for post-hepatectomy complications. The cut-off values were calculated by the sensitivity and 1-specificity. 
Figure 1

a) Total hepatectomy-related complications: Cut-off value: 2.5

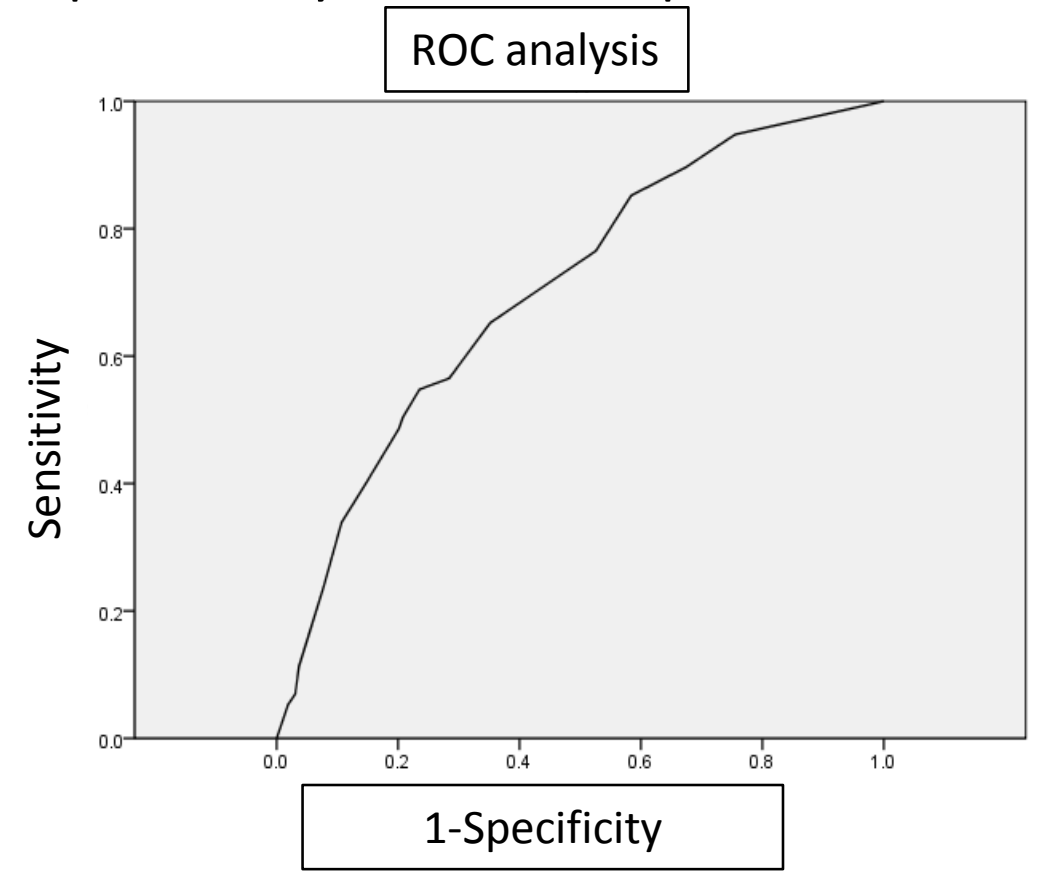

b) Uncontrolled ascites: Cut-off value: 3.0

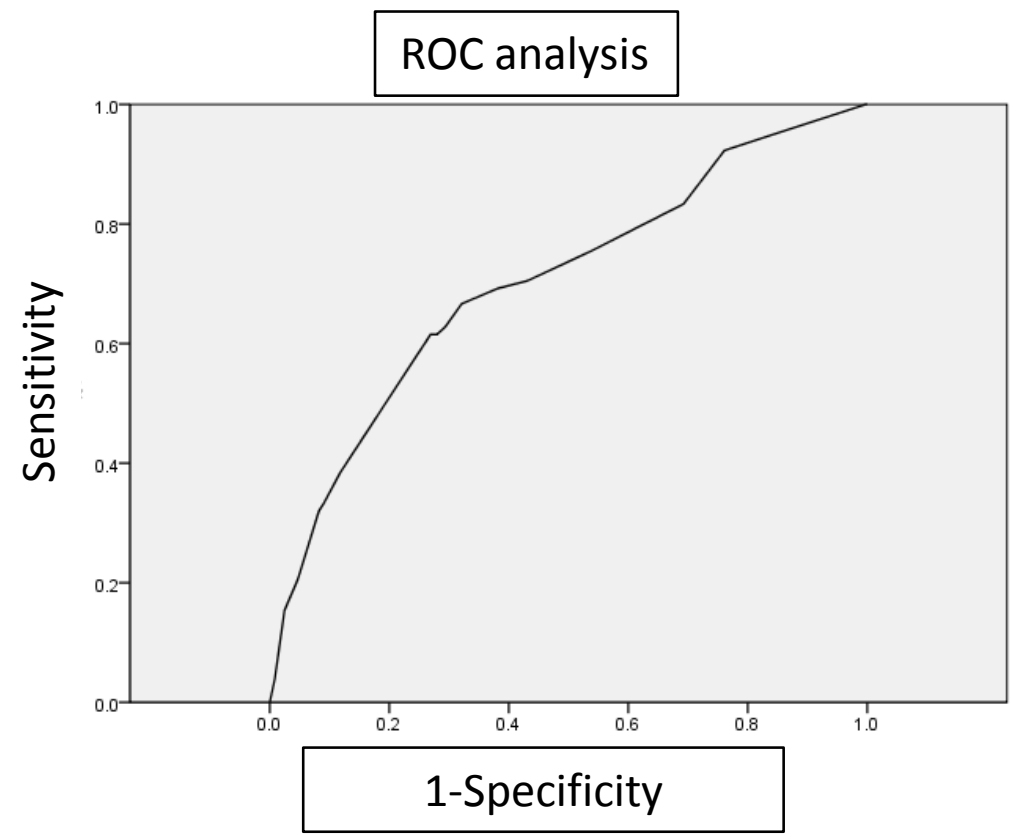


c) Intra-abdominal infection: Cut-off value: 5.7

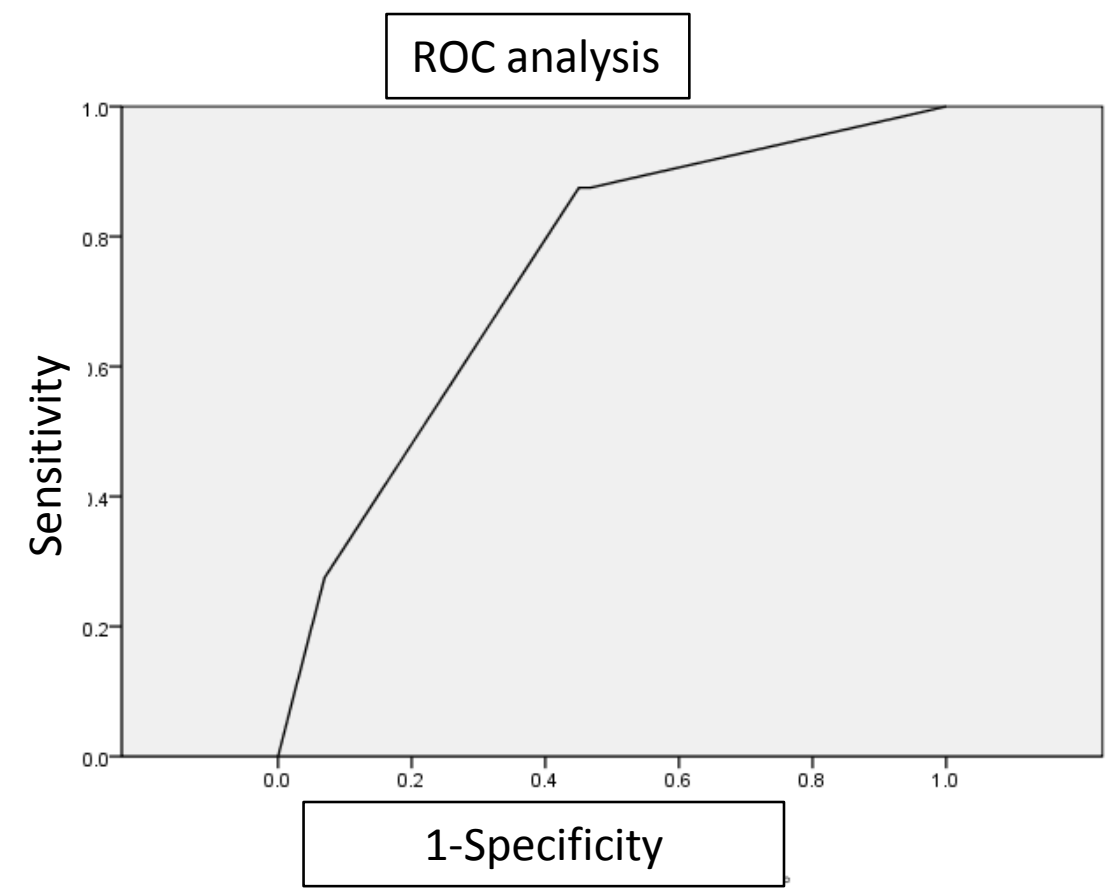

d) Hepatic failure: Cut-off value: 7.2

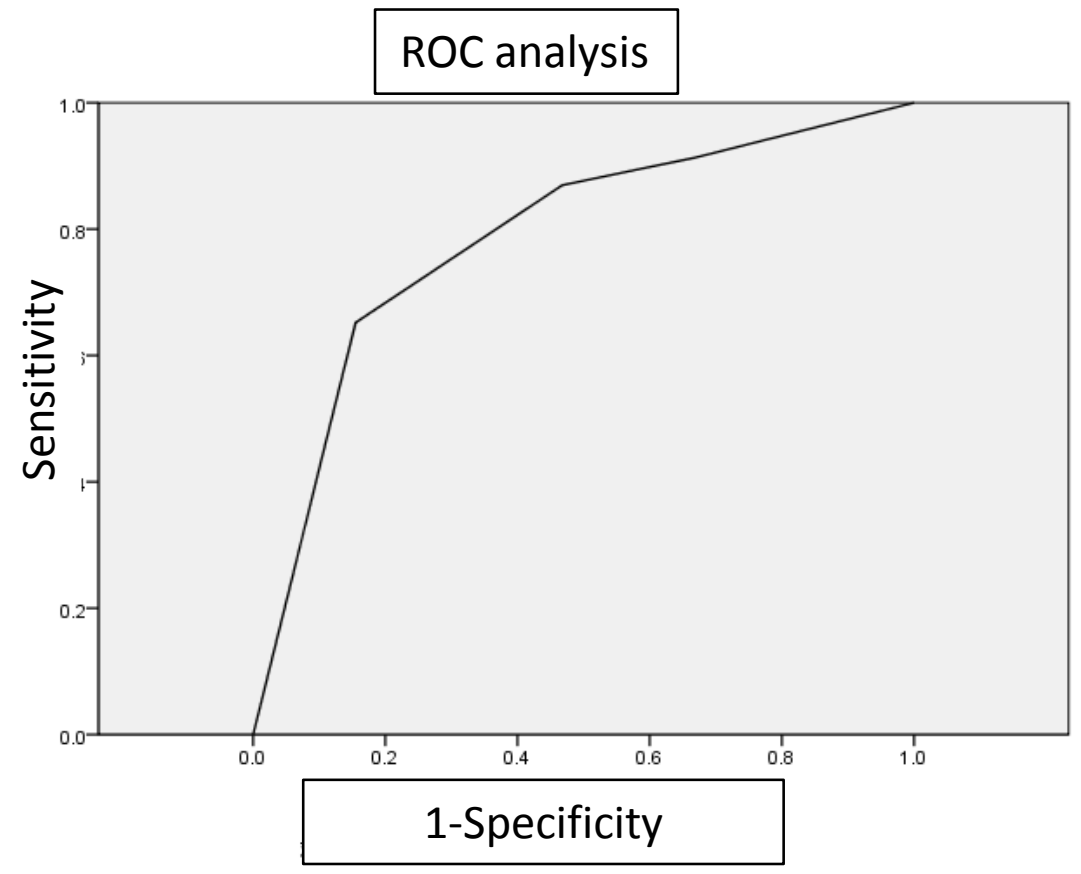


Table 1. Comparison of patient demographics, preoperative liver functional parameters and surgical records in patients with and without post-hepatectomy complications.

\begin{tabular}{|c|c|c|c|c|}
\hline & $\begin{array}{l}\text { Complication } \\
\qquad \begin{array}{c}(-) \\
(n=327)\end{array}\end{array}$ & $\begin{array}{l}\text { Complication } \\
\qquad \begin{array}{c}(+) \\
(n=115)\end{array}\end{array}$ & $\mathrm{P}$ value & $\begin{array}{c}\text { Predictive } \\
\text { cut-off value }\end{array}$ \\
\hline Age (years) & $66 \pm 11$ & $64 \pm 10$ & 0.057 & \\
\hline ICGR15 (\%) & $12.9 \pm 9.6$ & $15.2 \pm 9.3$ & 0.018 & 11 \\
\hline LHL15 & $0.92 \pm 0.03$ & $0.89 \pm 0.05$ & 0.015 & 0.90 \\
\hline HH15 & $0.58 \pm 0.08$ & $0.61 \pm 0.09$ & $<0.01$ & 0.60 \\
\hline LHL15/HH15 & $1.64 \pm 0.28$ & $1.53 \pm 0.29$ & $<0.01$ & 1.60 \\
\hline Total bilirubin (mg/dl) & $1.1 \pm 0.7$ & $1.4 \pm 1.8$ & 0.011 & 1.0 \\
\hline ALT (IU/L) & $58 \pm 64$ & $61 \pm 78$ & 0.445 & \\
\hline Platelet count $\left(/ \mathrm{mm}^{3}\right)$ & $20 \pm 9$ & $19 \pm 10$ & 0.128 & \\
\hline Prothrombin activity (\%) & $94 \pm 12$ & $91 \pm 16$ & 0.040 & 91 \\
\hline Hyaluronic acid (ng/ml) & $104 \pm 112$ & $196 \pm 188$ & $<0.01$ & 75 \\
\hline Operating time (minutes) & $412 \pm 128$ & $473 \pm 192$ & $<0.01$ & 500 \\
\hline Hepatic transection time (minutes) & $48 \pm 21$ & $50 \pm 21$ & 0.380 & \\
\hline
\end{tabular}


Blood loss (ml)

$922 \pm 879$

$1515 \pm 1052$

$<0.01$

950

Blood transfusion (ml)

$237 \pm 552$

$701 \pm 915$

$<0.01$

300

Data are presented as the mean \pm standard deviation.

Predictive value calculated by the analysis of receiver operating characteristic (ROC) curves.

ALT: alanine aminotransferase 
Table 2. Relationship between patient demographics or surgical factors and post-hepatectomy complications.

Complication (-) Complication (+) P value

$(n=327) \quad(n=115)$

Gender

Male/Female

212/115

$87 / 28$

0.088

Background liver

Normal/chronic hepatic injury/ obstructive jaundice

$169 / 143 / 15$

$33 / 68 / 14 \quad<0.01$

Liver Damage Grade

$\mathrm{A} / \mathrm{B}$

$296 / 31$

$79 / 36$

$<0.01$

Type of hepatectomy

Partial resection, subsegmentectomy or

205

53

0.003

segmentectomy

hemihepatectomy or more extended resection

122

62

Combined organ resection

No/ Yes

$251 / 76$

76/39

0.036

Combined biliary resection and anastomosis

No/ Yes

$269 / 58$

$88 / 27$

0.227

Combined major vascular resection

No/ Yes

$300 / 27$

$96 / 19$

0.019 
Use of vessel sealing devices during transection

No/ Yes

$227 / 100$

$87 / 28$

0.251

Liver Damage Grade using ICGR15 by the Liver Cancer Study Group of Japan [21] 
Table 3. Logistical multivariate regression analysis for risk factors associated with post-hepatectomy complications.

\begin{tabular}{|c|c|c|c|c|c|c|c|c|}
\hline & \multicolumn{2}{|c|}{$\begin{array}{c}\text { Hepatectomy associated } \\
\text { complications }\end{array}$} & \multicolumn{2}{|c|}{ Hepatic failure } & \multicolumn{2}{|c|}{ Uncontrolled ascites } & \multicolumn{2}{|c|}{$\begin{array}{l}\text { Intra-abdominal infections or } \\
\text { abscess formation }\end{array}$} \\
\hline & OR (95\% CI) & $P$ value & & & & & & \\
\hline $\begin{array}{l}\text { Chronic hepatic injury } \\
\text { Yes / No }\end{array}$ & $1.92(1.11-3.33)$ & 0.018 & $1.13(0.39-3.26)$ & 0.820 & $1.95(1.04-3.67)$ & 0.038 & $0.81(0.35-1.87)$ & 0.619 \\
\hline $\begin{array}{l}\text { ICGR15 } \\
\qquad \geq 11 \text { vs. }<11 \%\end{array}$ & $0.81(0.49-1.35)$ & 0.418 & $0.73(0.27-1.96)$ & 0.537 & $0.87(0.49-1.53)$ & 0.617 & & 0.405 \\
\hline $\begin{array}{l}\mathrm{HH} 15 \\
\qquad \geq 0.60 \text { vs. }<0.60\end{array}$ & $2.70(1.07-6.78)$ & 0.034 & $3.25(1.10-10.69)$ & 0.045 & $2.13(0.78-5.79)$ & 0.137 & $2.18(0.43-11.11)$ & 0.347 \\
\hline LHL15 & & & & & & & & \\
\hline $\begin{array}{l}\quad<0.90 \text { vs. } \geq 0.90 \\
\text { LHL15/HH15 }\end{array}$ & $1.00(0.42-2.34)$ & 0.418 & $2.67(0.83-8.65)$ & 0.100 & $0.63(0.29-1.36)$ & 0.238 & $0.89(0.28-2.87)$ & 0.846 \\
\hline $\begin{array}{l}<1.6 \text { vs. } \geq 1.6 \\
\text { Total bilirubin }\end{array}$ & $0.57(0.24-1.37)$ & 0.206 & $0.88(0.32-2.14)$ & 0.335 & $0.80(0.30-2.14)$ & 0.658 & $0.36(0.08-1.67)$ & 0.190 \\
\hline $\begin{array}{l}\quad \geq 1.0 \text { vs. }<1.0 \\
\text { Prothrombin activity }\end{array}$ & $2.42(1.44-4.08)$ & $<0.01$ & $2.36(0.85-6.61)$ & 0.100 & $1.76(0.97-3.19)$ & 0.062 & $0.69(0.28-1.66)$ & 0.402 \\
\hline $\begin{array}{l}\quad<90 \text { vs. } \geq 91 \% \\
\text { Hyaluronic acid level }\end{array}$ & $0.91(0.54-1.50)$ & 0.703 & $0.59(0.21-1.62)$ & 0.305 & $0.83(0.46-1.50)$ & 0.544 & $1.43(0.67-3.03)$ & 0.353 \\
\hline $\begin{array}{l}\quad \geq 75 \mathrm{vs.}<75 \mathrm{ng} / \mathrm{ml} \\
\text { Extend of hepatectomy }\end{array}$ & 1.79 (1.07-2.99) & 0.025 & $1.21(0.43-3.43)$ & 0.716 & $2.70(1.51-4.85)$ & $<0.01$ & $1.89(0.87-4.09)$ & 0.105 \\
\hline $\begin{array}{l}\text { Major vs. minor } \\
\text { Combined resection of other } \\
\text { organs }\end{array}$ & $1.79(1.05-3.06)$ & 0.032 & 3.21 (1.06-9.72) & 0.038 & $1.08(0.58-1.99)$ & 0.815 & $1.10(0.50-2.40)$ & 0.811 \\
\hline $\begin{array}{l}\text { Yes / No } \\
\text { Combined vascular resection }\end{array}$ & 1.35 (0.77-2.39) & 0.289 & $1.71(0.62-4.74)$ & 0.299 & $2.33(1.22-4.46)$ & 0.010 & $1.50(0.70-3.24)$ & 0.298 \\
\hline $\begin{array}{l}\text { Yes/ No } \\
\text { Operation time }\end{array}$ & $1.41(0.68-2.89)$ & 0.353 & $2.79(0.89-8,74)$ & 0.077 & $1.49(0.66-3.38)$ & 0.332 & $2.43(1.02-5.78)$ & 0.045 \\
\hline$\geq 500$ vs. $<500$ minutes & $0.65(0.23-1.29)$ & 0.566 & $4.76(1.21-18.63)$ & 0.024 & $0.80(0.43-1.48)$ & 0.472 & $5.69(1.99-16.26)$ & $<0.01$ \\
\hline
\end{tabular}

Major hepatectomy included hemihepatectomy or more resections. 
Table 4. Multiple Linear regression stepwise method output using data of 442 Patients for hepatic complications.

\begin{tabular}{|c|c|c|c|c|c|}
\hline \multirow[t]{2}{*}{ Model } & \multicolumn{2}{|c|}{ Unstandardized coefficients } & \multirow{2}{*}{$\begin{array}{c}\text { Standardized coefficient } \\
\beta\end{array}$} & \multirow{2}{*}{ t value } & \multirow{2}{*}{$\begin{array}{l}\text { Significance } \\
\text { (p value) }\end{array}$} \\
\hline & $\beta$ & Standard error & & & \\
\hline Model 1 (Constant) & 0.041 & 0.037 & & 1.076 & 0.282 \\
\hline Presence of chronic & 0.071 & 0.045 & 0.082 & 1.614 & 0.107 \\
\hline \multicolumn{6}{|l|}{ hepatic injury } \\
\hline HH $15>0.60$ & 0.094 & 0.045 & 0.103 & 2.067 & 0.039 \\
\hline Total bilirubin & 0.168 & 0.046 & 0.169 & 3.694 & 0.00003 \\
\hline \multicolumn{6}{|l|}{ level $>1.0 \mathrm{mg} / \mathrm{dl}$} \\
\hline Serum HA & 0.114 & 0.045 & 0.126 & 2.537 & 0.012 \\
\hline \multicolumn{6}{|l|}{ level>75ng/ml } \\
\hline Major hepatectomy & 0.152 & 0.041 & 0.170 & 3.701 & 0.0002 \\
\hline Model 2 (Constant) & 0.065 & 0.034 & & 1.904 & 0.058 \\
\hline HH $15 * 100$ & 0.112 & 0.044 & 0.124 & 2.557 & 0.011 \\
\hline Total bilirubin level & 0.164 & 0.046 & 0.166 & 3.607 & 0.0004 \\
\hline Serum HA level & 0.135 & 0.041 & 0.159 & 3.117 & 0.002 \\
\hline Extend of & 0.142 & 0.041 & 0.159 & 3.490 & 0.0005 \\
\hline hepatectomy & & & & & \\
\hline
\end{tabular}

Dependent variable: Step 2 total. The standardized $\beta$ coefficient provides a measure of the contribution of each variable to the model. The $t$ and $p$ values provide an 
indication of the impact of each predictor variable. Abbreviations as in Table 1. 
Table 5. Multiple Linear regression stepwise method output using data of 442 Patients for uncontrolled ascites, intraabdominal infection and hepatic failure

\begin{tabular}{|c|c|c|c|c|c|}
\hline \multirow[t]{2}{*}{ 1)Uncontrolled ascites } & \multicolumn{2}{|c|}{ Unstandardized coefficients } & \multirow{2}{*}{$\begin{array}{c}\text { Standardized coefficient } \\
\beta\end{array}$} & \multirow{2}{*}{$\mathrm{t}$ value } & \multirow{2}{*}{$\begin{array}{l}\text { Significance } \\
\text { (p value) }\end{array}$} \\
\hline & $\beta$ & Standard error & & & \\
\hline Model 1 (Constant) & 0.030 & 0.031 & & 0.964 & 0.335 \\
\hline Presence of chronic & 0.082 & 0.039 & 0.107 & 2.101 & 0.036 \\
\hline \multicolumn{6}{|l|}{ hepatic injury } \\
\hline Total bilirubin & 0.087 & 0.040 & 0.101 & 2.207 & 0.029 \\
\hline \multicolumn{6}{|l|}{ level $>1.0 \mathrm{mg} / \mathrm{dl}$} \\
\hline Serum HA & 0.150 & 0.039 & 0.191 & 3.863 & 0.0001 \\
\hline \multicolumn{6}{|l|}{ level>75ng/ml } \\
\hline Combined resection & 0.112 & 0.042 & 0.129 & 2.690 & 0.007 \\
\hline \multirow[t]{2}{*}{ 2)Intraabdominal } & \multicolumn{2}{|c|}{ Unstandardized coefficients } & Standardized coefficient & & Significance \\
\hline & $\beta$ & Standard error & $\beta$ & & \\
\hline Model 1 (Constant) & 0.019 & 0.019 & & 1.001 & 0.317 \\
\hline Combined resection & 0.116 & 0.045 & 0.124 & 2.607 & 0.010 \\
\hline
\end{tabular}


Operating time

0.123

0.027

0.214

4.521

$<0.0001$

$>500$ minutes

\begin{tabular}{|c|c|c|c|c|c|}
\hline \multirow[t]{2}{*}{ 3) Hepatic failure } & \multicolumn{2}{|c|}{ Unstandardized coefficients } & \multirow{2}{*}{$\begin{array}{c}\text { Standardized coefficient } \\
\beta\end{array}$} & \multirow{2}{*}{ t value } & \multirow{2}{*}{$\begin{array}{l}\text { Significance } \\
\text { (p value) }\end{array}$} \\
\hline & $\beta$ & Standard error & & & \\
\hline Model 1 (Constant) & -0.022 & 0.017 & & -1.323 & 0.186 \\
\hline HH $15>0.60$ & 0.075 & 0.021 & 0.164 & 3.530 & 0.0005 \\
\hline Major hepatectomy & 0.038 & 0.023 & 0.085 & 1.640 & 0.102 \\
\hline Operation time & 0.064 & 0.023 & 0.143 & 2.785 & 0.006 \\
\hline \multicolumn{6}{|l|}{$>$ 500minutes } \\
\hline Model 2 (Constant) & -0.014 & 0.016 & & -0.842 & 0.400 \\
\hline HН $15 * 100$ & 0.072 & 0.021 & 0.157 & 3.391 & 0.0008 \\
\hline Total bilirubin level & 0.080 & 0.021 & 0.179 & 3.871 & 0.0001 \\
\hline
\end{tabular}

Dependent variable: Step 1 or 2 total. The standardized $\beta$ coefficient provides a measure of the contribution of each variable to the model. The $t$ and $p$ values provide an indication of the impact of each predictor variable. 